\title{
Trial shows partial caries removal is an effective technique in primary molars
}

\author{
Abstracted from \\ Franzon R, Guimarães LF, Magalhães CE, Haas AN, Araujo FB.
}

Outcomes of One-Step Incomplete and Complete Excavation in Primary Teeth: A 24-Month Randomized Controlled Trial. Caries Res 2014; 48: 376-383. [Epub ahead of print] PubMed PMID: 2473208

Address for correspondence: Renata Franzon, Faculdade de Odontologia, UFRGS, Rua Ramiro Barcelos, 2492 Porto Alegre, RS (Brazil). E-mail: renatafranzon@hotmail.com

\section{Question: In carious primary molars is partial caries removal (PCR) more effective than total caries removal (TCR)?}

Design Randomised controlled trial in a university setting. Intervention Children aged three to eight years, with at least one molar with an acute, deep carious lesion into the dentine were recruited. Treatment took place under rubber dam with decayed dentine being removed completely from the lateral walls of cavities in both groups using round burs operated at low speed. TCR or PCR was then performed in the pulpal wall of each tooth. After caries removal teeth were restored with calcium hydroxide cement and composite resin. Teeth with pulpal exposure were pulpotomised using ferric sulphate.

Outcome measure The presence of a fistula, swelling, spontaneous pain and mobility not compatible with root resorption were considered to be clinical signs of failure. Radiolucency at the furcation or in the periapical region and internal or external pathological resorption were considered to be radiographic signs of failure. Results One hundred and twenty-four teeth in 51 patients were randomised. In the TCR group there were 57 teeth and 38 patients, with 41 patients and 67 teeth in the PCR group. Three patients (four teeth; one PCR and three TCR) dropped out leaving 120 teeth (PCR: $n$ = 66; TCR: $\mathrm{n}=54$ ) for analysis. In the TCR group $27.5 \%$ (15) teeth in 13 children had pulp exposure compared with one tooth in one child in the PCR group (2\%). The mean operative time was significantly higher for TCR (28.1 min; 95\% Cl: 23.6-32.6 min) than for PCR (17.9 min; $95 \% \mathrm{Cl}: 16.3-19.5 \mathrm{~min})$. There was no statistical difference in success rates at 24 months between the groups. The success rate in the TCR group was 96\%; (95\% Cl: 85-99\%) compared with 92\%; (95\% Cl: 81-96\%) in the PCR group.

Conclusions The clinical and radiographic success rates of PCR and TCR in primary teeth with deep carious lesions were high and did not differ significantly, indicating that PCR is a reliable minimally invasive approach in primary teeth and that the retention of carious dentine does not interfere with pulp vitality. Moreover, PCR provided other clinically relevant advantages over TCR, especially lower incidence of pulp exposure and lower operative time.

\section{Commentary}

There is growing evidence supporting the use of more conservative techniques to treat carious primary and permanent teeth. Current caries management for deep lesions includes a variety of approaches; two-step caries removal (stepwise excavation), where incomplete caries removal is carried out at the first appointment then at the second visit the remaining caries is removed to give complete caries removal, then sealing; one-step caries excavation, where incomplete caries removal is followed immediately by placement of a definitive restoration (partial caries removal); and techniques that involve no caries removal and sealing. ${ }^{1}$

So far, studies looking at incomplete caries removal have shown advantages for the more conservative caries removal techniques to treat deep, cavitated carious lesions over complete caries removal. ${ }^{1,2}$ The main advantages are the significant reduction of pulp exposure accompanied by no detriment to the tooth in terms of pulpal symptoms.

This study by Franzon et al. overall reinforces previous studies' findings, comparing the two-year clinical and radiographic outcomes of one-step partial caries removal (PCR) and total caries removal (TCR) performed in primary molars with deep caries 'inner quarter of dentine assessed radiographically' of three to eight year olds. The mean incidence of pulp exposure was significantly lower in PCR (2\%) than TCR (27.5\%; p<0.01). Clinically, overall the majority of treated teeth (94\%), independent of the treatment performed, showed no failures after one year. By comparing the success rates among the two treatment groups no statistical benefit of one approach over another was observed $(\mathrm{p}=0.34)$, even when treatments in multisurface lesions were compared to occlusal lesions ( $\mathrm{p}=0.08)$.

The methodology of this RCT is acceptable. The main limitation of this study is around the subjective criteria assessing how much carious tissue was removed. Although the authors state that PCR was carried out to maintain much of the decayed tissue, excavation was stopped when a 'leathery' consistence was observed. This leads us to assume that most of the infected tissue was removed leaving only a thin layer of infected dentine above the pulp. By this criterion, it is possible that caries was completely removed in some areas of the pulpal wall creating a pulp exposure even in the partial caries removal group where the aim of the treatment is to avoid this.

An unrestricted allocation was carried out; however, it was not clear who allocated treatments or if allocation was at treatment time, a possible source of selection bias in this study. On the other 


\section{CARIES}

hand, the use of standardised clinical procedures including local anaesthetic, rubber dam and identical filling materials (composite) across both arms for clinical procedures, increased the quality of the study and reduced the risk of performance bias. Patients and examiners were masked to the intervention; however, masking of operators (as with most dental procedures) was not possible.

Despite increasing numbers of clinical trials looking at comparisons between incomplete/no caries removal, there is still no agreement amongst researchers as to what constitutes partial caries removal (there were still pulp exposures in the PCR arm) and so how do we translate these findings to clinical practice? Exactly how much caries should be left to avoid pulp exposures and still achieve the best long-term outcomes for our patients? Issues around terminology and standardisation of interventions need to be addressed. Having said that, although there is some risk of bias, this study adds to the growing picture of evidence that in asymptomatic primary teeth with deep caries, PCR reduces the incidence of pulp exposure and is not harmful compared to TCR. It is also a more efficient use of operative time. These findings support PCR as a clinical and timeeffective technique in the management of dentinal carious lesions in primary molars.

\section{Practice points}

- Dental practitioners should consider the use of more conservative techniques like partial caries excavation over total caries excavation for patients presenting with, symptomless, deep carious primary teeth, as it reduces pulp exposure and it does not have any harmful effect on the teeth

- The use of partial caries removal reduces significantly the operative time during treatment of deep carious lesions. This could be advantageous for treatment of paediatric patients.

Ruth Santamariaa, Nicola Innesb

aDepartment of Preventive and Pediatric Dentistry, Ernst-MoritzArndt University of Greifswald, Greifswald, Germany ${ }^{b}$ Division of Oral Health Sciences, School of Dentistry, University of Dundee, Scotland, UK

1. Ricketts D, Lamont T, Innes NP, Kidd E, Clarkson JE. Operative caries management in adults and children. Cochrane Database Syst Rev 2013; 3: CD003808.

2. Schwendicke F, Dörfer CE, Paris S. Incomplete caries removal: a systematic review and meta-analysis. J Dent Res 2013; 92: 306-314.

Evidence-Based Dentistry (2014) 15, 81-82. doi:10.1038/sj.ebd.6401044 